\title{
Intermolecular dynamics studied by paramagnetic tagging
}

\author{
Xingfu Xu • Peter H. J. Keizers • \\ Wolfgang Reinle $\cdot$ Frank Hannemann • \\ Rita Bernhardt · Marcellus Ubbink
}

Received: 12 December 2008/Accepted: 23 February 2009/Published online: 10 March 2009

(C) The Author(s) 2009. This article is published with open access at Springerlink.com

\begin{abstract}
Yeast cytochrome $c$ and bovine adrenodoxin form a dynamic electron transfer complex, which is a pure encounter complex. It is demonstrated that the dynamic nature of the interaction can readily be probed by using a rigid lanthanide tag attached to cytochrome $c$. The tag, Caged Lanthanide NMR Probe 5, induces pseudocontact shifts and residual dipolar couplings and does not perturb the binding interface. Due to the dynamics in the complex, residual dipolar couplings in adrenodoxin are very small. Simulation shows that cytochrome $c$ needs to sample a large part of the surface of adrenodoxin to explain the small degree of alignment observed for adrenodoxin. The applied method provides a simple and straightforward way to observe dynamics in protein complexes or domain-domain mobility without the need for external alignment media.
\end{abstract}

Keywords Dynamics - Lanthanide tag ·

Encounter complex · Residual dipolar couplings ·

Paramagnetic NMR

Abbreviations
RDC $\quad$ Residual dipolar coupling
C $c \quad$ Cytochrome $c$

Electronic supplementary material The online version of this article (doi:10.1007/s10858-009-9308-0) contains supplementary material, which is available to authorized users.

X. Xu · P. H. J. Keizers · M. Ubbink $(\square)$

Institute of Chemistry, Leiden University, P.O. Box 9502,

2300 RA Leiden, The Netherlands

e-mail: m.ubbink@chem.leidenuniv.nl

W. Reinle $\cdot$ F. Hannemann $\cdot$ R. Bernhardt

Naturwissenschaftlich-Technische Fakultät III,

Institut für Biochemie, Universität des Saarlandes,

P.O. Box 151150, 66041 Saarbrücken, Germany
Adx
Adrenodoxin
PCS
Pseudocontact shift
CLaNP-5
Caged Lanthanide NMR Probe 5
PRE
Paramagnetic relaxation enhancement

\section{Introduction}

Protein dynamics is important for protein function and recognition. Solution NMR spectroscopy is a powerful tool to study protein dynamics on a timescale ranging from pico-seconds to seconds. ${ }^{15} \mathrm{~N}$ spin relaxation measurements combined with Liparo-Szabo analysis is now a standard NMR method to characterize the protein dynamics on the ps-ns timescale (Palmer 2004). Recently, relaxation dispersion has been developed as the method to characterize the protein dynamics in the $\mu \mathrm{s}-\mathrm{ms}$ time scale (Korzhnev and Kay 2008). Residual dipolar couplings (RDCs) were initially proposed as a tool to provide long-range restraints for structure refinement. The RDC is also sensitive to motions spanning the ps-ms timescale. Thus, the dynamics information obtained from RDCs offer a view on dynamic processes that is complementary to other NMR spectroscopic approaches (Tolman and Ruan 2006; Lakomek et al. 2008; Lange et al. 2008).

RDCs can be measured when biomolecules are slightly aligned with the external magnetic field. For some metalloproteins, weak molecular alignment can be achieved at high fields due to their anisotropic magnetic susceptibility (Tolman et al. 1995; Banci et al. 1998). For other proteins, external alignment media have to be used (Tjandra et al. 1997; Tjandra and Bax 1997; Bax and Grishaev 2005). The interpretation of RDC data in the study of intermolecular dynamics is difficult when an external alignment medium is used, because the alignment tensors of the two molecules 
will be different for each relative orientation and the information obtained is only relative to the external magnetic field (Tolman and Ruan 2006). In the study of bimolecular interaction involving nucleic acids, the problem can be overcome by an elegant method in which an elongated RNA is used to obtain alignment, providing a fixed reference frame for RDC analysis (Zhang et al. 2007). For protein-protein complexes or protein domain-domain interactions with two rigid substructures, preferential alignment of one component and detection of RDCs on the other provides a more direct way for analysis of intermolecular dynamics, see Fig. 1 (Bertini et al. 2004; Rodriguez-Castañeda et al. 2006).

Lanthanide paramagnetic probes offer an attractive way to achieve such partial alignment (Otting 2008). Two-point attachment of the paramagnetic probe Caged Lanthanide NMR Probe 5 (CLaNP5) through cysteine disulfide bridges proved to be a good way to introduce large and welldefined paramagnetic effects by minimizing the dynamics of probe (Keizers et al. 2007; Keizers et al. 2008).

In this study, CLaNP-5 is used to study the intermolecular dynamics in the complex of yeast cytochrome $c$ (Cc) and bovine adrenodoxin (Adx). Adx is involved in steroid hormone biosynthesis by acting as an electron shuttle between NADPH-dependent adrenodoxin reductase and several cytochromes P450 (Grinberg et al. 2000). In vitro electron transfer from adrenodoxin reductase to Adx is often monitored by fast subsequent electron transfer from Adx to mitochondrial $\mathrm{C} c$. Yeast $\mathrm{C} c$ is used as a model of P450 and the non-physiological complex between yeast $\mathrm{C} c$ and bovine $\mathrm{Adx}$ is active in electron transfer. It is interesting that electron transfer in this

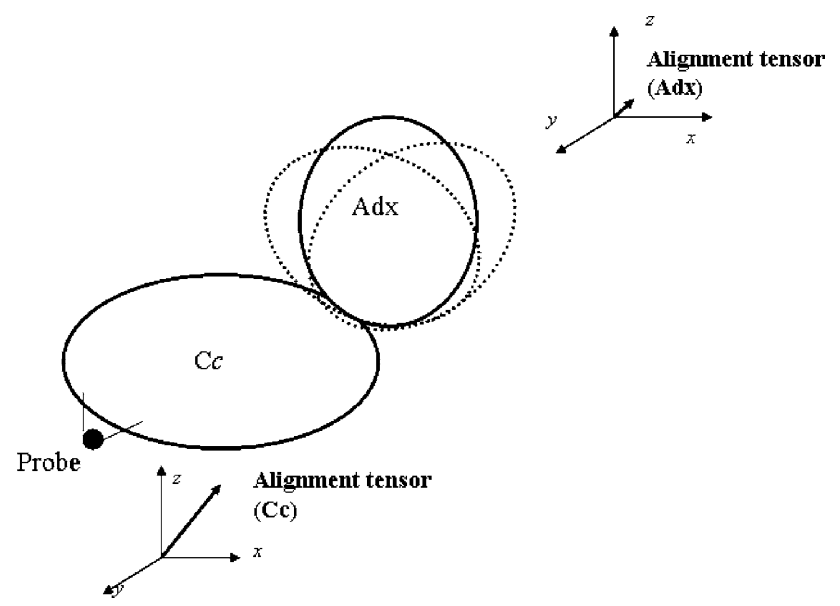

Fig. 1 Schematic representation of the method used in this study. A paramagnetic probe CLaNP-5 is rigidly attached to the backside of $\mathrm{C} c$ through two arms. At high magnetic field, $\mathrm{C} c$ is slightly aligned. The alignment effect on Adx is dramatically reduced due to conformational averaging complex is so rapid, because it is a non-physiological complex. Many complexes of electron transfer proteins have evolved to exhibit fast turn-over and show little specificity. For example, for the physiological complexes of cytochrome $c$ and cytochrome $c$ peroxidase (Volkov et al. 2006) and myoglobin and cytochrome $b_{5}$ (Worrall et al. 2002; Liang et al. 2004) it has been shown that the encounter state, which by nature is an ensemble of nonspecific, electrostatic complexes, represents a significant or even dominant fraction of the entire complex. A previous study on the complex of $\mathrm{C} c$ and Adx showed that it too is very dynamic and can be considered as a pure encounter complex (Xu et al. 2008) and yet it is active in electron transfer. The study of the amplitude of the dynamic motions, i.e. the degree of surface sampling, will help to understand how such non-specific electron transfer complexes can still be active.

Large RDCs are observed for $\mathrm{C} c$ when CLaNP-5 is attached. In the complex, very small RDCs are detected for Adx, suggesting averaging over multiple orientations of $\mathrm{Adx}$ relative to $\mathrm{C} c$ due to large intermolecular dynamics.

\section{Materials and methods}

\section{Site-directed mutagenesis}

Mutant CcN56C/L58C was created by site-specific mutagenesis with the overlap extension method (Ho et al. 1989). The template DNA used for mutagenesis was pUC-cc containing the genes of yeast iso-1 $\mathrm{C} c(\mathrm{C} 102 \mathrm{~T})$ and $\mathrm{C} c$ heme lyase. The following four primers were used in three PCRs to amplify the mutated insert; M13 forward (5'-CCCAGTCAC GACGTTGTAAAACG-3'), M13 reverse primer (5'-AGCG GATAACAATTTCACACAGG-3'), N56C/L58C forward primer (5'-GCCAAT ATCAAGAAATGCGTGTGCTGGG ACG-3') and N56C/L58C backward primer (5'-GTCCCAG CACACGCATTTCTTGATATTGGC- $3^{\prime}$ ). The product of the third PCR was purified and subcloned into the XmaI/ HindIII site of the plasmid pUC-18. The DNA sequence of pUC-ccN56C/L58C was verified by sequencing.

\section{Protein production and ${ }^{15} \mathrm{~N}$ labeling}

The double mutant $\mathrm{C} c \mathrm{~N} 56 \mathrm{C} / \mathrm{L} 58 \mathrm{C}$ was produced and purified using the same protocol as for wild type $\mathrm{Cc}(\mathrm{Xu}$ et al. 2008). The final yield was $15 \mathrm{mg} \mathrm{L}^{-1}$ and $8 \mathrm{mg} \mathrm{L}^{-1}$ for the unlabeled and ${ }^{15} \mathrm{~N}$ labeled $\mathrm{C} c$ mutant, respectively. The concentration of $\mathrm{C} c$ mutant was determined using an extinction coefficient $27.5 \mathrm{mM}^{-1} \mathrm{~cm}^{-1}$ at $550 \mathrm{~nm}$ for the reduced $\mathrm{C} c$ mutant. The production of both unlabeled and ${ }^{15} \mathrm{~N}$ labeled Adx has been described (Worrall et al. 2003). 
Paramagnetic probe attachment

The synthesis of Ln-CLaNP-5 probe has been described (Keizers et al. 2008). To attach Ln-CLaNP-5 to CcN56C/

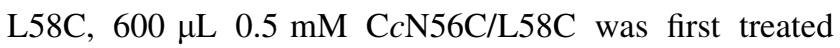
with DTT (final concentration $5 \mathrm{mM}$ ) at room temperature for $2 \mathrm{hrs}$ to remove possible dimers by reduction of disulfide bonds. A PD-10 column (GE Healthcare) was used to remove the DTT and bring the protein in the $3 \mathrm{~mL}$ buffer containing $20 \mathrm{mM}$ sodium phosphate and $150 \mathrm{mM}$ $\mathrm{NaCl}, \mathrm{pH}$ 7.0. Seven to ten equivalents CLaNP-5 were added and the solution was stirred for $2 \mathrm{~h}$ at $4^{\circ} \mathrm{C}$. The buffer was changed to $20 \mathrm{mM}$ sodium phosphate, $\mathrm{pH} 7.0$ before the solution was loaded on a HiTrap-SP column. The protein was eluted using a $350-700 \mathrm{mM} \mathrm{NaCl}$ gradient in $20 \mathrm{mM}$ sodium phosphate, $\mathrm{pH}$ 7.0. Monomeric $\mathrm{C} c$ attached to CLaNP-5 was concentrated and one equivalent of ascorbate was added to keep the $\mathrm{C} c$ in reduced state. Either ytterbium or lutetium containing CLaNP-5 was attached to the $\mathrm{C} c$ double mutant. The extent of probe attachment to ${ }^{15} \mathrm{~N} \mathrm{CcN56C/L58C}$ was estimated to be above $90 \%$ from mass spectrometry and from the absence of diamagnetic crosspeaks in the HSQC spectra of the paramagnetic samples.

\section{NMR spectroscopy}

All NMR samples contained $20 \mathrm{mM}$ potassium phosphate buffer, $\mathrm{pH} 7.4,6 \% \mathrm{D}_{2} \mathrm{O}$ and one equivalent of ascorbic acid under an argon atmosphere to keep $\mathrm{C} c$ in the reduced state. Three sets of samples were prepared comprising free ${ }^{15} \mathrm{~N}$ $\mathrm{C} c$ with probe attached and the CLaNP-Cc/Adx $(0.25 \mathrm{mM}$ 1:1) complex in which either $\mathrm{C} c \mathrm{~N} 56 \mathrm{C} / \mathrm{L} 58 \mathrm{C}$ or Adx was ${ }^{15} \mathrm{~N}$ labeled. $\mathrm{Yb}-\mathrm{CLaNP}-5$ was used in the paramagnetic samples and Lu-CLaNP-5 acted as a diamagnetic control. For pseudocontact shift measurements, ${ }^{1} \mathrm{H}-{ }^{15} \mathrm{~N}$ HSQC spectra were recorded at $14.1 \mathrm{~T}$ on a Bruker DMX $600 \mathrm{MHz}$ spectrometer equipped with a TCI-Z-GRAD cryoprobe operating at $293 \mathrm{~K}$. For ${ }^{1} \mathrm{H}_{-}{ }^{15} \mathrm{~N}$ RDC measurements, ${ }^{1} \mathrm{H}-{ }^{15} \mathrm{~N}$ IPAP-HSQC (Ottiger et al. 1998) spectra were acquired at $21.1 \mathrm{~T}$ on a Bruker $900 \mathrm{MHz}$ spectrometer equipped with a TCI-Z-GRAD cryoprobe. The RDC was obtained through the measurement of the splitting difference in the ${ }^{15} \mathrm{~N}$ doublet of amide resonances between the samples containing paramagnetic $\mathrm{Yb}$ and diamagnetic Lu. NMR data were processed in Azara (www. bio.cam.ac.uk/azara), and analyzed in Ansig for Windows (Helgstrand et al. 2000).

PCS and RDC analysis

The crystal structure of yeast iso-1 Cc (PDB entry: 1YCC (Louie and Brayer 1990)) was used for PCS and RDC analysis. Hydrogens were added in XPLOR-NIH (Schwieters et al. 2003) according to the standard geometry parameters. A set of pseudo-atoms representing the magnetic susceptibility tensor frame was added for the fit of PCSs and RDCs using PARAstraints in XPLOR-NIH (Banci et al. 2004). The equation used for the tensor calculation is

$\mathrm{PCS}=\frac{1}{12 \pi r^{3}}\left[\Delta \chi_{a x}\left(3 \cos ^{2} \theta-1\right)+\frac{3}{2} \Delta \chi_{r h} \sin ^{2} \theta \cos 2 \Omega\right]$

where $r, \theta$, and $\Omega$ are the polar coordinates of the nucleus with respect to the principal axes of the $\chi$-tensor and $\Delta \chi_{\text {ax }}$ and $\Delta \chi_{\mathrm{rh}}$ are the axial and rhombic components of the $\chi$-tensor, respectively. The position of the lanthanide was determined by energy minimization of the PCS energy term and a term that restrained the metal to 6-10 $\AA$ from the $\mathrm{C} \alpha$ of cysteines 56 and 58 of $\mathrm{C} c$ (Keizers et al. 2008). The lowest-energy structure was used for fitting of RDCs using the FRUN algorithm in the PARAstraints suite. The equation used for RDC alignment tensor determination is

$$
\begin{aligned}
D^{\mathrm{res}}= & -\frac{B_{0}^{2}}{15 k T} \frac{\gamma_{i} \gamma_{j} h}{16 \pi^{3} r_{i j}^{3}} \\
& \left(\Delta \chi_{a x}\left(3 \cos ^{2} \theta-1\right)+\frac{3}{2} \Delta \chi_{r h} \sin ^{2} \theta \cos 2 \Omega\right)
\end{aligned}
$$

where $B_{0}$ is the applied magnetic field, $\gamma_{i}$ and $\gamma_{j}$ are the gyromagnetic ratios of nuclei $i$ and $j$ respectively, $r_{i j}$ is the $i-j$ inter-nuclear distance (1.02 $\AA$ for amide $\mathrm{N}-\mathrm{H}$ bonds) (Jeffrey 1992), $\theta$ and $\Omega$ determine the inter-nuclear vector orientation with respect to the principle axes of the $\chi$-tensor, $T$ is the absolute temperature, and $k$ and $h$ are the Boltzmann and Planck constants, respectively.

\section{Simulation of the dynamics}

The coordinates of the $\mathrm{Ln}-\mathrm{C} c$ structure and Adx (PDB entry 1AYF (Müller et al. 1998)) were used for simulations to explore the minimal sampling space required for averaging of the intermolecular RDCs. Similar to what was described before (Xu et al. 2008), the relative diffusional movement of the proteins in the native complex was decomposed into two types of rotations. One is the rotation of Adx around its own center of mass (wobbling motion) and the other is the rotation of $\mathrm{Adx}$ around the $\mathrm{C} c$, resulting in the translational movement over its surface. The heme metal center of $\mathrm{C} c$ was used as the rotating center for the latter movement. Each rotation can be further decomposed into the rotations around the $\mathrm{X}, \mathrm{Y}$ and $\mathrm{Z}$ axes. Six angle variables are thus used to describe the rotation ranges. The initial orientation of two proteins was generated in a way that N16 of $\mathrm{C} c$ is in close contact with L80 of Adx. The initial distance of the geometrical center of $\mathrm{Adx}$ to the 
metal alignment center was set to $42 \AA$. This brought the proteins together in an orientation consistent with chemical shift perturbation data. The effect of averaging over the six rotating and wobbling axes was evaluated by generating 100 sterically allowed orientations randomly within a given angle range. The averaged intermolecular RDCs were calculated for amide protons of Adx in this ensemble and compared with the observed RDCs for Adx in the complex. By stepwise increments of the angle ranges the amplitude of motion in the simulation was increased. By varying the ranges of the six angle variables independently, the contribution to each of them to the averaging effect could be established.

\section{Results}

Probe attachment on $\mathrm{C} c$

Two surface-exposed residues of Cc, N56 and L58 were mutated to cysteines for probe attachment. These residues are located in the region of a short $\beta$-strand (V57-W59), at the backside of $\mathrm{C} c$ with respect to the heme edge, which is the site involved in binding to various interaction partners (Ubbink and Bendall 1997; Worrall et al. 2001; Crowley et al. 2002; Banci et al. 2003; Volkov et al. 2005). The attachment of the probe to a site far from the interaction interface should cause little perturbation of the complex. The side chains of the two residues are 5-10 ̊ apart, an optimal distance for attachment of the two arms of the CLaNP probe. Via two disulfide bridges formed between the cysteine residues and the arms of the Ln-probe, the latter was rigidly attached to the protein surface. The molecular weight of the purified product of $\mathrm{Lu}-\mathrm{CLaNP}{ }^{15} \mathrm{~N}$ $\mathrm{C} c(13547.9 \pm 1 \mathrm{Da})$ agrees with the theoretical molecular weight (13548.2 Da), confirming that both leaving groups are removed and the probe is attached via two disulphide bridges. The yield of the entire labeling procedure, including purification is about $50 \%$.

\section{Magnetic susceptibility tensor determination}

The ${ }^{15} \mathrm{~N}-\mathrm{HSQC}$ of $\mathrm{Lu}-\mathrm{C} c$ is similar to that of wt $\mathrm{C} c$ except for a few residues in close proximity to the sites of mutation, indicating that probe attachment does not cause large perturbations of the structure. For $\mathrm{Yb}-\mathrm{C} c$ PCSs were caused by the interaction between the unpaired electrons of the lanthanide ion and the nuclei of $\mathrm{C} c$ and were measured by comparison of HSQC spectra for samples with diamagnetic $\mathrm{Lu}$ and paramagnetic $\mathrm{Yb}-\mathrm{CLaNP}-5$. A single set of PCSs was observed for $\mathrm{Yb}-\mathrm{C} c$ as compared to $\mathrm{Lu}-\mathrm{C} c$. For each backbone amide ${ }^{1} \mathrm{H}$ and ${ }^{15} \mathrm{~N}$ pair far from the metal site, similar PCSs are expected (in ppm) for the ${ }^{1} \mathrm{H}$ and ${ }^{15} \mathrm{~N}$ nuclei.
This leads to the unambiguous assignment of some backbone amides at the initial stage, allowing magnetic susceptibility tensor determination and subsequent assignment of more residues in an iterative procedure. The largest PCS ( $>2 \mathrm{ppm})$ was detected for the amide proton of W59 of Cc (Fig. 2 and Table S1). In total, 73 amide protons of reduced $\mathrm{C} c$ with probe attached were assigned and used for magnetic susceptibility tensor $(\Delta \chi)$ determination by fitting to the crystal structure of yeast $\mathrm{C} c$ (1YCC). The sizes of the axial and rhombic components $\left(\Delta \chi_{\mathrm{ax}}\right.$ and $\Delta \chi_{\mathrm{rh}}$ in $\left.10^{-32} \mathrm{~m}^{3}\right)$ are $8.3 \pm$ 0.2 and $3.1 \pm 0.2$ respectively. Figure 3 shows a correlation plot of experimentally observed and theoretically predicted PCS. The good fit ( $Q$ factor: 0.047) supports the finding that CLaNP is rigid relative to the frame of the protein.

Probe induced RDC

At $21.1 \mathrm{~T}$ RDCs as large as $9 \mathrm{~Hz}$ were detected for $\mathrm{C} c$ attached to the $\mathrm{Yb}$-probe (Table S2). The alignment tensor was determined using $64 \mathrm{RDCs}$ of $\mathrm{N}-\mathrm{H}$ pairs of $\mathrm{C} c$. Figure $4 \mathrm{a}$ shows the correlation for experimental and back-calculated RDCs by fitting to the crystal structure of $\mathrm{C} c$ (Q factor 0.263 ). The correlation is acceptable given the error margins $(1.5 \mathrm{~Hz}$ for this $23 \mathrm{kDa}$ complex) and the fact that the crystal structure was not refined using the RDC data. The fitted alignment tensor is 6.71 and 2.0 (in $10^{-32} \mathrm{~m}^{3}$ ) for axial and rhombic components, respectively. The size of the axial component derived from RDC data is 19\% smaller than the one from the PCSs. The correlation between observed RDCs and RDCs

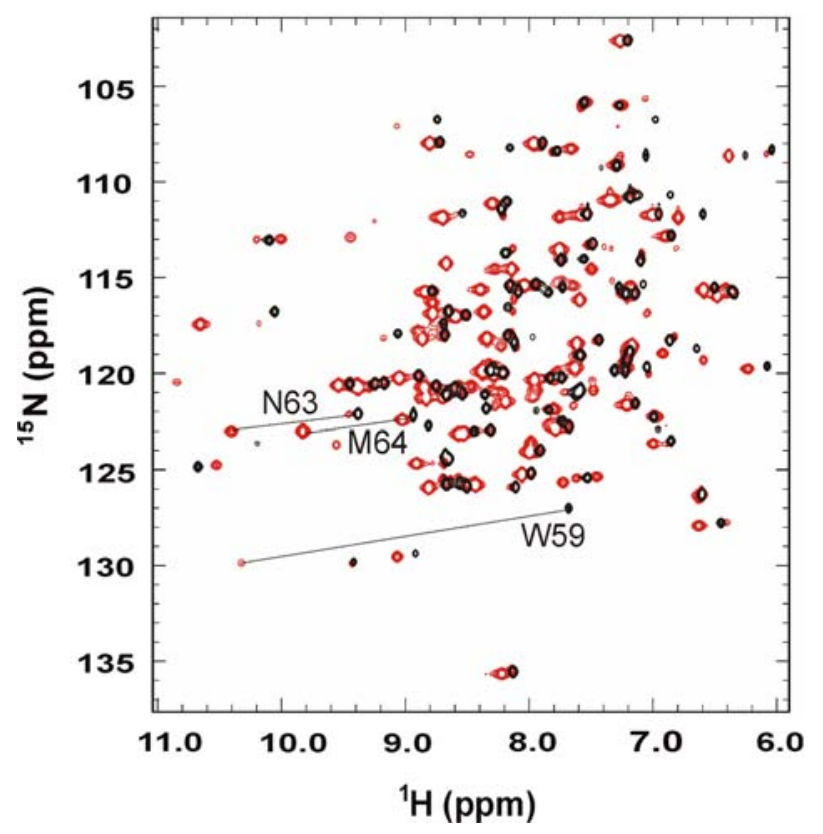

Fig. 2 Overlay of ${ }^{15} \mathrm{~N}-{ }^{1} \mathrm{H}$ HSQC spectra of ${ }^{15} \mathrm{~N}$-labeled $\mathrm{C} c \mathrm{~N} 56 \mathrm{C} /$ L58C attached to Yb-CLaNP-5 (red) or Lu-CLaNP-5 (Black). The spectra were recorded at $293 \mathrm{~K}$ and $14.1 \mathrm{~T}$ 


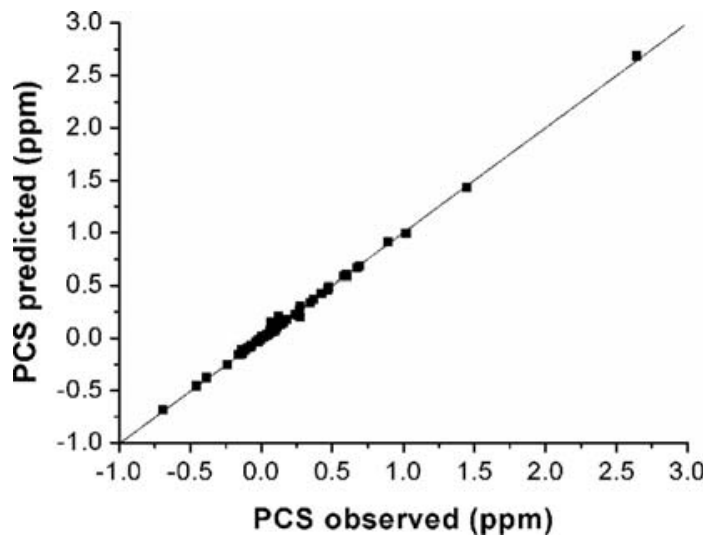

Fig. 3 Back-predicted PCSs plotted versus experimentally determined PCSs of backbone amide protons of $\mathrm{C} c$ attached with CLaNP-5. The solid line indicates a perfect correlation

predicted with the PCSs derived tensor is shown in Fig. 4b. The $\mathrm{Q}$ factor for this fit is 0.287 , only slightly larger than that of the fit based on the RDC derived tensor. Thus, it could be that the difference between the $\Delta \chi$ values derived from RDCs and PCSs is not significant. Alternatively, the discrepancy could be attributable to internal dynamics, because RDCs are more sensitive to the internal dynamics than PCSs. Such dynamics may represent slight mobility of the probe, but can also be caused by mobility of amide groups (Bouvignies et al. 2005).

The interaction of $\mathrm{Ln}-\mathrm{C} c$ with Adx

The interface of $\mathrm{Ln}-\mathrm{C} c$ determined from chemical shift perturbations is similar to that in the native complex (Table S3 in supplementary material). A titration experiment of unlabeled $\mathrm{Adx}$ to ${ }^{15} \mathrm{~N} \mathrm{Lu}-\mathrm{C} c$ yielded a $K_{\mathrm{a}}$ value 2.1 $( \pm 0.7) \times 10^{4} \mathrm{M}^{-1}$ for binding of $\mathrm{Ln}-\mathrm{C} c$ to Adx (Fig. 5). This value is slightly smaller than that for the wild type Cc/Adx complex, $4.0 \pm 1.0 \times 10^{4} \mathrm{M}^{-1}$ (Worrall et al. 2003), possibly due to the small temperature difference of the measurements. The NMR titration for the complex with wt $\mathrm{C} c$ was conducted at $285 \mathrm{~K}$, whereas $293 \mathrm{~K}$ was used in the present study. Using the ${ }^{15} \mathrm{~N}$ labeled $\mathrm{C} c$ attached to CLaNP-5, both PCSs and RDCs were measured for $\mathrm{C} c$ in the complex Cc/Adx. In the 1:1 complex of CLaNP5$\mathrm{C} c /$ Adx, the observed PCSs and RDCs for $\mathrm{C} c$ are the weighted average of free and complex states. A comparison of PCSs and RDCs for free $\mathrm{Yb}-\mathrm{C} c$ and $\mathrm{Yb}-\mathrm{C} c$ in the complex (Tables S1 and S2) indicates that the magnetic susceptibility tensor does not change upon complex formation.

Small paramagnetic effects on Adx in the Cc/Adx complex

When Ln-Cc interacts with ${ }^{15} \mathrm{~N}$ Adx, the paramagnetic effects observed for Adx are proportional to the fraction of Adx bound in the complex. At the ratio of $1: 1 \mathrm{Adx} / \mathrm{Ln}-\mathrm{C} c$ $(0.25 \mathrm{mM}), 61 \% \mathrm{Adx}$ is in the complex state. A few residues of Adx in the 1:1 complex show small intermolecular PCSs. An attempt to model the orientation of two proteins with these PCSs failed. According to the Eq. 1, only small PCSs are expected for a few residues of Adx even in the singleorientation complex because the Ln metal center is relatively far $(>35 \AA)$ from most of the residues of Adx. RDCs on the other hand are independent of the amide-Ln distance. If the degree of alignment of Adx in the complex is the same as for $\mathrm{Yb}-\mathrm{C} c$, RDCs of $4-5 \mathrm{~Hz}$ are expected, and when extrapolated to the $100 \%$ bound state, values up to $9 \mathrm{~Hz}$ are predicted. The largest extrapolated RDC observed for Adx in the $100 \%$ bound state is about $2 \mathrm{~Hz}$ (Fig. 6), which is much smaller than the RDCs determined for $\mathrm{C} c$ and below the significance level $(1.5 \mathrm{~Hz}$ error margin $\times 1 / 0.61$ for extrapolation to $100 \%$ bound $=2.5 \mathrm{~Hz}$ ). The striking difference of RDC values detected for Adx and $\mathrm{C} c$ in the complex is in accord with the intermolecular dynamics between $\mathrm{C} c$ and $\mathrm{Adx}$ in the complex.

\section{Modelling of the intermolecular dynamics}

A simulation was performed to explore the magnitude of the intermolecular motions in the complex. The movement
Fig. 4 Plots of experimental and back-predicted RDCs. a Experimental RDCs of $\mathrm{Yb}-\mathrm{Cc}$ at $21.1 \mathrm{~T}$ vs. RDCs backcalculated using the RDC based alignment tensor.

b Experimental RDCs of $\mathrm{Yb}-\mathrm{C} c$ vs. RDCs back-calculated using the tensor determined with PCSs. The solid and dashed lines represent the perfect correlation and the $1.5 \mathrm{~Hz}$ error margins, respectively
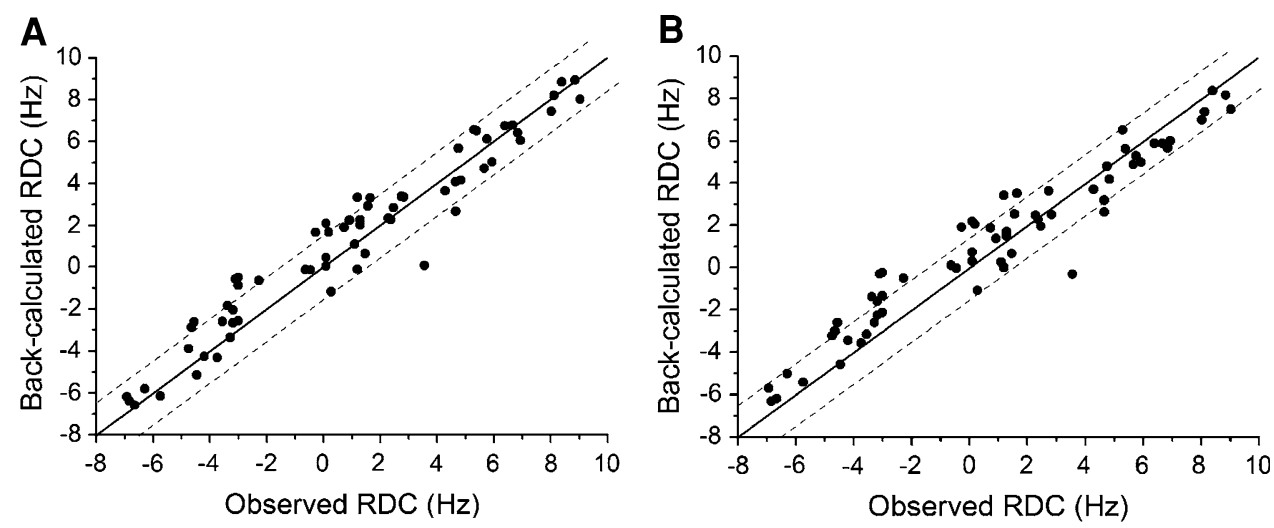


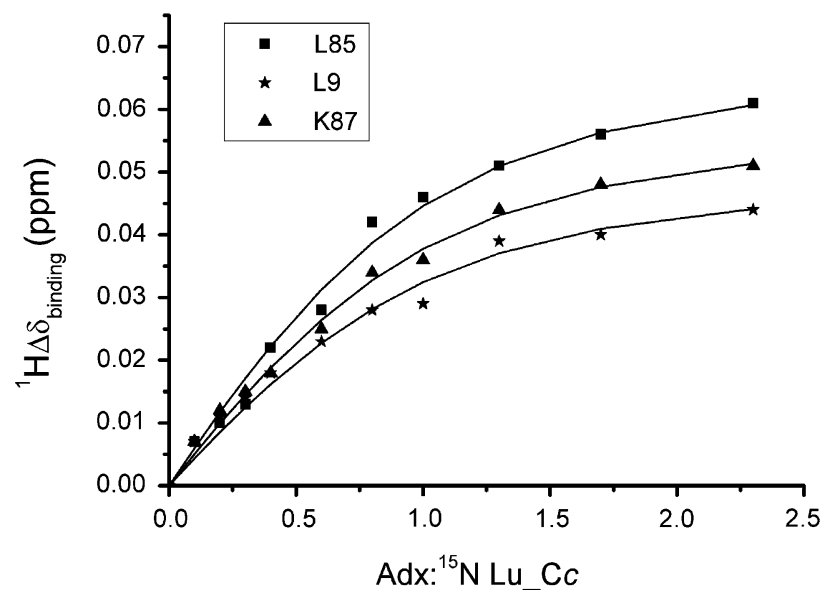

Fig. 5 Amide chemical shift perturbation curves of ${ }^{15} \mathrm{~N} \mathrm{Lu}-\mathrm{C} c$ titrated with Adx. Chemical shift changes $\left({ }^{1} \mathrm{H} \Delta \delta_{\text {binding }}\right)$ of residues of ${ }^{15} \mathrm{~N} \mathrm{Lu}-\mathrm{C} c$ are plotted against the Adx: ${ }^{15} \mathrm{~N} \mathrm{Lu}-\mathrm{C} c$ molar ratio. A $K_{a}$ of $2.1 \pm 0.7 \times 10^{4} \mathrm{M}^{-1}$ is obtained from a global fit to a 1:1 binding model (Worrall et al. 2003)
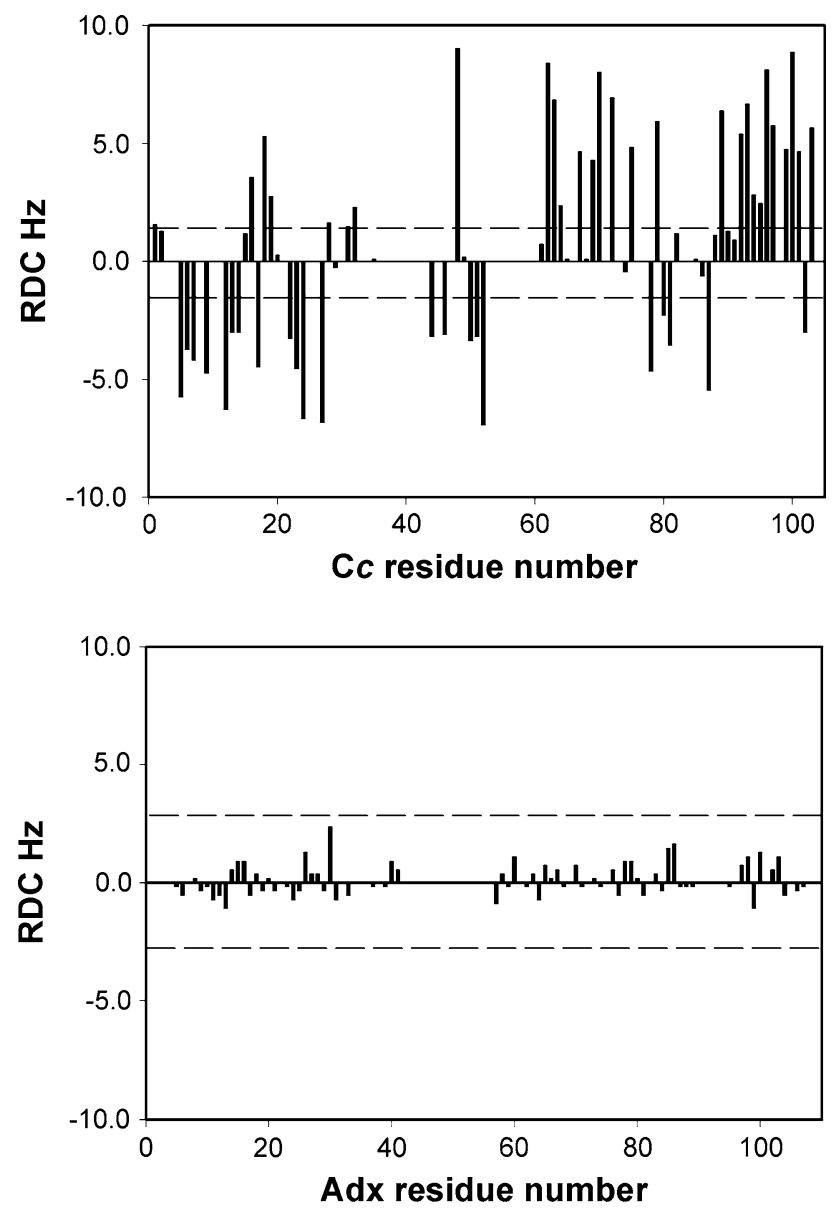

Fig. 6 Comparison of RDCs detected on $\mathrm{C} c$ and Adx. Upper panel: $\mathrm{RDCs}$ detected for $\mathrm{Yb}-\mathrm{C} c$, lower panel: RDCs observed for Adx in the complex $\mathrm{Yb}-\mathrm{C} c / \mathrm{Adx}$ (extrapolated to $100 \%$ bound). The dashed lines indicate the error margins for $\mathrm{C} c(1.5 \mathrm{~Hz})$ and the $100 \%$ bound $\operatorname{Adx}(2.5 \mathrm{~Hz})$. The data were acquired at $21.1 \mathrm{~T}$ of the proteins within the complex was decomposed into six different rotations (see Materials and methods section). Within a set sampling space, all sterically allowed conformations were randomly sampled. In this way, ensembles of the protein complexes were generated and compared with the observed chemical shift perturbations and RDCs. In any single-orientation conformation of $\mathrm{Ln}-\mathrm{C} c / \mathrm{Adx}$, the predicted largest RDCs for amide protons of Adx are as large as $\pm 8-9 \mathrm{~Hz}$, similar to those detected for $\mathrm{C} c$. The insignificant RDCs observed for Adx in the complex are the direct result of intermolecular dynamics, which averages the $\mathrm{N}-\mathrm{H}$ bond orientations relative to the reference frame. The simulation suggests that the rotation of $\mathrm{Adx}$ around $\mathrm{C} c$ (translational movement of $\mathrm{Adx}$ over the $\mathrm{C} c$ surface) hardly reduces the averaged RDC values on Adx (Figure S1 in supplementary material). This is in contrast to the large averaging effect of the same movement on PCS originating from heme center in the previous study $\mathrm{Xu}$ et al. 2008). The rotation of Adx around its own geometrical center can lead to a large change of $\mathrm{N}-\mathrm{H}$ inter-nuclear vector orientation ( $\theta$ and $\Omega$ in Eq. 2 ) with respect to the principal axes of the magnetic susceptibility tensor, thus averaging the RDC to an insignificant value. In an ensemble with a set of maximal rotation angles of 90, 120 and 40 degrees of rotations around the geometrical center of $\operatorname{Adx}$ for $\mathrm{x}, \mathrm{y}$ and $\mathrm{z}$ rotations, respectively, the intermolecular RDC can be averaged out. In this ensemble, $\mathrm{C} c$ needs to sample a large surface area of Adx (Fig. 7a). The sampling space in this ensemble agrees well with the chemical shift perturbation data and is also consistent with the degree of dynamics suggested by our previous study using both PCSs derived from the $\mathrm{C} c$ heme and paramagnetic relaxation enhancement (PRE) mapping from spin labeling (Xu et al. 2008).

\section{Discussion}

Paramagnetic metal ions provide NMR effects such as PRE, PCS and RDC due to self-orientation at high magnetic field. These effects can be converted into distance or angular restraints which are useful not only for structure determination, but also for the study of dynamics. PRE is isotropic and inversely dependent on the metal-nucleus distance $\left(1 / r^{6}\right.$ dependence). Therefore, PRE is powerful to detect minor populations in a heterogeneous system (Volkov et al. 2006; Tang et al. 2006; Tang et al. 2007). PCS depends on the metal-nucleus orientation and distance, falling off with $r^{3}$. The magnitude of metal-induced RDCs is not dependent on metal-nucleus distance, thus each of these observables provides a different view on the dynamics in the system. PCSs and RDCs exhibit averaging for all motions occurring on a time scale faster than $\sim 10^{-2} \mathrm{~s}$. 

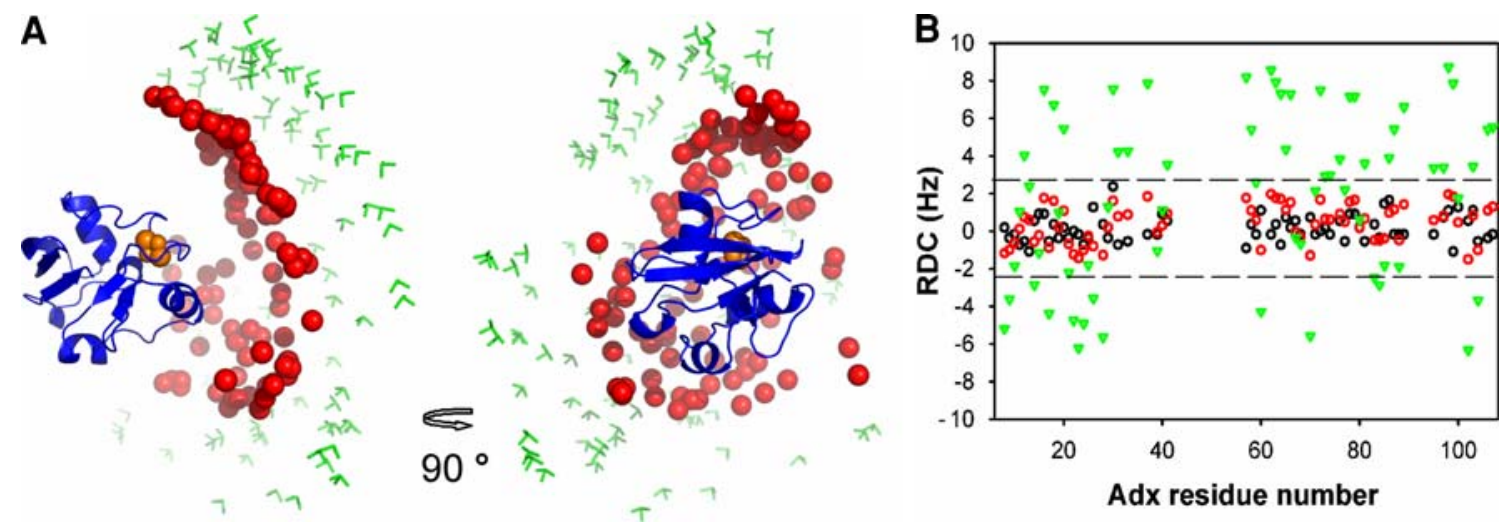

Fig. 7 The ensemble of the Cc/Adx complexes from RDC simulation illustrates the intermolecular dynamics. a Adx is shown as a blue ribbon. The FeS cluster is shown as orange spheres. The geometrical centers of $\mathrm{C} c$ are represented by red spheres. The tensor frame experienced for each conformation of $\mathrm{C} c$ is represented as green sticks. b Plots of RDCs observed for Adx in the complex and the

For a two-component system (two domains or proteins) aligned with an external medium, the presence of intermolecular dynamics may be induced from differences between the alignment tensors experienced by the two components (Braddock et al. 2002; Ulmer et al. 2002). Failure to observe such a difference, however, does not exclude the possibility of dynamics. If the components tumble entirely independently the two tensors are only determined by shape or charge properties of each of the two components. If, on the other hand, the components interact, both tensors will differ for each relative conformation of the two components. Contrary to this, metal-induced alignment provides a fixed reference alignment frame for one component, enabling direct detection of motions of the other component. This method already proved useful to obtain information on dynamics of domain-domain interactions of metallo-proteins (Bertini et al. 2004, 2007). To extend this method to other proteins without metal sites, a paramagnetic tag needs to be attached rigidly to the protein surface.

CLaNP-5 has been shown to exhibit minimized internal dynamics through attachment via two disulfides bridges (Keizers et al. 2007, 2008). In this study, the Yb-CLaNP-5 was rigidly attached to the backside of reduced, diamagnetic $\mathrm{C} c$ to obtain large PCSs and RDCs, with much smaller paramagnetic effects observed for $\mathrm{Adx}$ in the dynamic complex. Among all Ln metals, Yb is classified as moderately paramagnetic, with a small broadening effect. More highly paramagnetic Ln metals (Dy, Tb, Tm) which induce larger PCSs and RDCs (Keizers et al. 2008), can provide more extensive datasets for accurately modeling the 3-D sampling space of the dynamic complex. Although in this study two metalloproteins were involved, this method should be generally applicable to other protein complexes or multi-domain proteins without metal-binding
RDCs simulated for the Adx in the dynamic ensemble. Black and red circles represent observed and calculated RDC respectively. Green triangles represent calculated $\mathrm{RDC}$ for $\mathrm{Adx}$ in a single-orientation complex. The dashed lines indicate the significance level, $2.5 \mathrm{~Hz}$ for the $100 \%$ bound $\mathrm{Adx}$

sites, because the heme and FeS cluster were not used in this study in any way.

Acknowledgements Financial support was provided by the Fonds der chem. Industrie (R. Bernhardt), the Volkswagenstiftung (R. Bernhardt, M. Ubbink, P.H.J. Keizers, and X. Xu, grant I/80854) and the Netherlands Organisation for Scientific Research (M. Ubbink and $\mathrm{X}$. Xu, grants 700.50.026 and 700.52.425). RDC measurements were carried out on a $900 \mathrm{MHz}$ spectrometer in the NMR facility of the Bijvoet Center in Utrecht and Dr H. Wienk is acknowledged for the technical help on NMR measurements. H. van der Elst is acknowledged for assistance with MS measurements.

Open Access This article is distributed under the terms of the Creative Commons Attribution Noncommercial License which permits any noncommercial use, distribution, and reproduction in any medium, provided the original author(s) and source are credited.

\section{References}

Banci L, Bertini I, Huber JG, Luchinat C, Rosato A (1998) Partial orientation of oxidized and reduced cytochrome $b_{5}$ at high magnetic fields: magnetic susceptibility anisotropy contributions and consequences for protein solution structure determination. J Am Chem Soc 120:12903-12909

Banci L, Bertini I, Felli IC, Krippahl L, Kubicek K, Moura JJG, Rosato A (2003) A further investigation of the cytochrome $b_{5}$-cytochrome $c$ complex. J Biol Inorg Chem 8:777-786

Banci L, Bertini I, Cavallaro G, Giachetti A, Luchinat C, Parigi G (2004) Paramagnetism-based restraints for Xplor-NIH. J Biomol NMR 28:249-261

Bax A, Grishaev A (2005) Weak alignment NMR: a hawk-eyed view of biomolecular structure. Curr Opin Struct Biol 15:563-570

Bertini I, Del Bianco C, Gelis I, Katsaros N, Luchinat C, Parigi G, Peana M, Provenzani A, Zoroddu MA (2004) Experimentally exploring the conformational space sampled by domain reorientation in calmodulin. Proc Natl Acad Sci USA 101:6841-6846

Bertini I, Gupta YK, Luchinat C, Parigi G, Peana M, Sgheri L, Yuan J (2007) Paramagnetism-based NMR restraints provide maximum 
allowed probabilities for the different conformations of partially independent protein domains. J Am Chem Soc 129:12786-12794

Bouvignies G, Bernadó P, Blackledge M (2005) Protein backbone dynamics from N-H-N dipolar couplings in partially aligned systems: a comparison of motional models in the presence of structural noise. J Magn Reson 173:328-338

Braddock DT, Louis JM, Baber JL, Levens D, Clore GM (2002) Structure and dynamics of $\mathrm{KH}$ domains from FBP bound to single-stranded DNA. Nature 415:1051-1056

Crowley PB, Rabe KS, Worrall JAR, Canters GW, Ubbink M (2002) The ternary complex of cytochrome $f$ and cytochrome $c$ : identification of a second binding site and competition for plastocyanin binding. Chembiochem 3:526-533

Grinberg AV, Hannemann F, Schiffler B, Müller J, Heinemann U, Bernhardt R (2000) Adrenodoxin: structure, stability, and electron transfer properties. Proteins 40:590-612

Helgstrand M, Kraulis P, Allard P, Härd T (2000) Ansig for Windows: an interactive computer program for semiautomatic assignment of protein NMR spectra. J Biomol NMR 18:329-336

Ho SN, Hunt HD, Horton RM, Pullen JK, Pease LR (1989) Sitedirected mutagenesis by overlap extension using the polymerase chain-reaction. Gene 77:51-59

Jeffrey G (1992) Single-crystal neutron diffractometry. In: Domenicano A, Hargittai I (eds) Accurate molecular structure, their determination and importance. Oxford University Press, Oxford, pp 270-298

Keizers PHJ, Desreux JF, Overhand M, Ubbink M (2007) Increased paramagnetic effect of a lanthanide protein probe by two-point attachment. J Am Chem Soc 129:9292-9293

Keizers PH, Saragliadis A, Hiruma Y, Overhand M, Ubbink M (2008) Design, synthesis, and evaluation of a lanthanide chelating protein probe: CLaNP-5 yields predictable paramagnetic effects independent of environment. J Am Chem Soc 130:14802-14812

Korzhnev DM, Kay LE (2008) Probing invisible, low-populated states of protein molecules by relaxation dispersion NMR spectroscopy: an application to protein folding. Acc Chem Res 41:442-451

Lakomek NA, Walter KFA, Farés C, Lange OF, de Groot BL, Grubmüller H, Brüschweiler R, Munk A, Becker S, Meiler J, Griesinger C (2008) Self-consistent residual dipolar coupling based model-free analysis for the robust determination of nanosecond to microsecond protein dynamics. J Biomol NMR 41:139-155

Lange OF, Lakomek NA, Farés C, Schroder GF, Walter KFA, Becker S, Meiler J, Grubmüller H, Griesinger C, de Groot BL (2008) Recognition dynamics up to microseconds revealed from an RDC-derived ubiquitin ensemble in solution. Science 320:14711475

Liang ZX, Kurnikov IV, Nocek JM, Mauk AG, Beratan DN, Hoffman BM (2004) Dynamic docking and electron-transfer between cytochrome $b_{5}$ and a suite of myoglobin surface-charge mutants. Introduction of a functional-docking algorithm for proteinprotein complexes. J Am Chem Soc 126:2785-2798

Louie GV, Brayer GD (1990) High-resolution refinement of yeast iso1-cytochrome $c$ and comparisons with other eukaryotic cytochromes $c$. J Mol Biol 214:527-555

Müller A, Müller J, Müller Y, Uhlman H, Bernhard R, Heineman (1998) New aspects of electron transfer revealed by the crystal structure of a truncated bovine adrenodoxin, $\operatorname{Adx}(4-108)$. Structure 6:269-280

Ottiger M, Delaglio F, Bax A (1998) Measurement of J and dipolar couplings from simplified two-dimensional NMR spectra. J Magn Reson 131:373-378
Otting G (2008) Prospects for lanthanides in structural biology by NMR. J Biomol NMR 42:1-9

Palmer AG (2004) NMR characterization of the dynamics of biomacromolecules. Chem Rev 104:3623-3640

Rodriguez-Castañeda F, Haberz P, Leonov A, Griesinger C (2006) Paramagnetic tagging of diamagnetic proteins for solution NMR. Magn Reson Chem 44:S10-S16

Schwieters CD, Kuszewski JJ, Tjandra N, Clore GM (2003) The Xplor-NIH NMR molecular structure determination package. J Magn Reson 160:65-73

Tang C, Iwahara J, Clore GM (2006) Visualization of transient encounter complexes in protein-protein association. Nature 444:383-386

Tang C, Schwieters CD, Clore GM (2007) Open-to-closed transition in apo maltose-binding protein observed by paramagnetic NMR. Nature 449:1078-1082

Tjandra N, Bax A (1997) Direct measurement of distances and angles in biomolecules by NMR in a dilute liquid crystalline medium. Science 278:1111-1114

Tjandra N, Omichinski JG, Gronenborn AM, Clore GM, Bax A (1997) Use of dipolar $1 \mathrm{H}-15 \mathrm{~N}$ and $1 \mathrm{H}-13 \mathrm{C}$ couplings in the structure determination of magnetically oriented macromolecules in solution. Nat Struct Biol 4:732-738

Tolman JR, Ruan K (2006) NMR residual dipolar couplings as probes of biomolecular dynamics. Chem Rev 106:1720-1736

Tolman JR, Flanagan JM, Kennedy MA, Prestegard JH (1995) Nuclear magnetic dipole interactions in field-oriented proteins information for structure determination in solution. Proc Natl Acad Sci USA 92:9279-9283

Ubbink M, Bendall DS (1997) Complex of plastocyanin and cytochrome c characterized by NMR chemical shift analysis. Biochemistry 36:6326-6335

Ulmer TS, Werner JM, Campbell ID (2002) SH3-SH2 domain orientation in Src kinases: NMR studies of Fyn. Structure 10:901-911

Volkov AN, Ferrari D, Worrall JAR, Bonvin AMJJ, Ubbink M (2005) The orientations of cytochrome $c$ in the highly dynamic complex with cytochrome $b_{5}$ visualized by NMR and docking using HADDOCK. Protein Sci 14:799-811

Volkov AN, Worrall JAR, Holtzmann E, Ubbink M (2006) Solution structure and dynamics of the complex between cytochrome $c$ and cytochrome $c$ peroxidase determined by paramagnetic NMR. Proc Natl Acad Sci USA 103:18945-18950

Worrall JAR, Kolczak U, Canters GW, Ubbink M (2001) Interaction of yeast iso-1-cytochrome $c$ with cytochrome $c$ peroxidase investigated by $\left[{ }^{15} \mathrm{~N},{ }^{1} \mathrm{H}\right]$ heteronuclear NMR spectroscopy. Biochemistry 40:7069-7076

Worrall JAR, Liu Y, Crowley PB, Nocek JM, Hoffman BM, Ubbink M (2002) Myoglobin and cytochrome $b_{5}$ : a nuclear magnetic resonance study of a highly dynamic protein complex. Biochemistry 41:11721-11730

Worrall JAR, Reinle W, Bernhardt R, Ubbink M (2003) Transient protein interactions studied by NMR spectroscopy: the case of cytochrome $c$ and adrenodoxin. Biochemistry 42:7068-7076

$\mathrm{Xu}$ XF, Reinle WG, Hannemann F, Konarev PV, Svergun DI, Bernhardt R, Ubbink M (2008) Dynamics in a pure encounter complex of two proteins studied by solution scattering and paramagnetic NMR spectroscopy. J Am Chem Soc 130:63956403

Zhang Q, Stelzer AC, Fisher CK, Al-Hashimi HM (2007) Visualizing spatially correlated dynamics that directs RNA conformational transitions. Nature 450:1263-1267 